\section{Decentralised HIV care during the COVID-19 pandemic: Ensuring safe community-based services}

To the Editor: Since the start of the COVID-19 pandemic, there have been significant concerns about disruptions to the continuum of HIV care worldwide. ${ }^{[1-6]}$ Expansion of differentiated service delivery has been proposed as one strategy to maintain safe access to HIV care, ${ }^{[7]}$ with increased use of differentiated services reported during the early stages of the COVID-19 pandemic. ${ }^{[8]}$ Decentralised HIV care delivery programmes providing out-of-facility care, in particular, decrease frequency of visits to healthcare facilities, ${ }^{[7]}$ minimising exposures to COVID-19 and allowing continuity of HIV care during lockdowns and other restrictions. However, providing decentralised, out-offacility HIV care during the pandemic requires access to COVID-19 information, capacity to screen patients for COVID-19 symptoms and adequate supply of personal protective equipment at all points of care. Supporting staff and ensuring access to these resources may be more difficult in community-based sites.

We sought to evaluate perceptions of personal and facility preparedness among healthcare workers at primary health clinics and community-based pick-up points participating in a decentralised antiretroviral therapy (ART) delivery programme in KwaZuluNatal, South Africa. The Central Chronic Medicines Dispensing and Distribution programme allows stable, virologically suppressed patients to collect ART at community-based pick-up points, such as private pharmacies and churches. ${ }^{[9]}$ We administered a telephone questionnaire to a convenience sample of staff in primary health clinics during April and May 2020 and staff in clinics and community-based pick-up points in August 2020 to assess access to COVID-19 information, available resources and perceived personal and facility preparedness.

We completed interviews with 112 clinic staff ( $n=49$ in April May 2020; $n=63$ in August 2020) and 24 pick-up-point staff. Most clinic staff were healthcare providers (e.g. nurses, medical officers) or ancillary providers (e.g. counsellors), while pick-up-point staff were mainly pharmacists and dispensing clerks. Respondents primarily perceived an increased need for prevention procedures and supplies (59\%) as a challenge to their work during April - May, and COVID19 infection risk (48\%) in August. By August 2020, nearly all clinic staff reported access to resources (94\%) and space (100\%) to screen patients for COVID-19, while significantly fewer pick-up-point staff reported access to these tools (54\%; $p<0.001)$. In August 2020, pick-up-point staff, compared with clinic staff, reported significantly lower rates of access to information to perform work duties during the COVID-19 pandemic (67\% v. 94\%; $p=0.003)$, perceived facility preparedness to work with COVID-19 patients ( $54 \%$ v. $81 \%$; $p=0.016$ ) and access to the necessary protective equipment ( $50 \%$ v. $89 \% ; p<0.001)$. Our results suggest that staff working in out-of-facility, communitybased sites participating in decentralised HIV care programmes do not feel as prepared to work with patients during the COVID-19 pandemic, or do not have access to the necessary supplies to carry out this work safely, compared with their clinic-based counterparts. While further study is needed, our results highlight that if differentiated service delivery is to fulfil its potential to ensure continuity of care for people living with HIV during the COVID-19 pandemic, staff at all programme levels and sites must be appropriately supported and prepared to deliver care safely to their patients.

Acknowledgements. None.

Author contributions. All authors contributed to the study conception and design. Material preparation, data collection and analysis were performed by JJ, JY, SG, BAB, NN, ZMS, ARK, GN, LMN, NJW, DZ, RAP and IVB. The first draft was written by JJ, and all authors commented on previous versions of the manuscript. All authors read and approved the final document. Funding. This work was funded by the National Institutes of Health (NIH): T32AI007433 (JJ), R01 MH114997 (IVB), K24 AI141036 (IVB), and the Weissman Family MGH Research Scholar Award (IVB). Its contents are solely the responsibility of the authors and do not necessarily represent the official views of the NIH or the Massachusetts General Hospital Executive Committee on Research.

Conflicts of interest. None.

\section{Jana Jarolimova}

Massachusetts General Hospital, Division of Infectious Diseases, Boston; and Medical Practice Evaluation Center, Boston, USA

jjarolimova@partners.org

\section{Joyce Yan}

Massachusetts General Hospital, Biostatistics Center, Boston, USA

\section{Sabina Govere}

AIDS Healthcare Foundation, Durban, South Africa

\section{Bridget A Bunda}

Massachusetts General Hospital, Medical Practice Evaluation Center, Boston, USA

Nompumelelo Ngobese, Zinhle M Shazi, Anele R Khumalo, Gugulami Nelson, Lungile M Ngcobo

AIDS Healthcare Foundation, Durban, South Africa

\section{Nafisa J Wara, Danielle Zionts}

Massachusetts General Hospital, Medical Practice Evaluation Center, Boston, USA

\section{Robert A Parker}

Massachusetts General Hospital, Biostatistics Center, Boston; Harvard University, Center for AIDS Research, Boston; and Harvard Medical School, Boston, USA

\section{Ingrid V Bassett}

Massachusetts General Hospital, Division of Infectious Diseases, Boston; Medical Practice Evaluation Center, Boston; Harvard University, Center for AIDS Research, Boston; and Harvard Medical School, Boston, USA

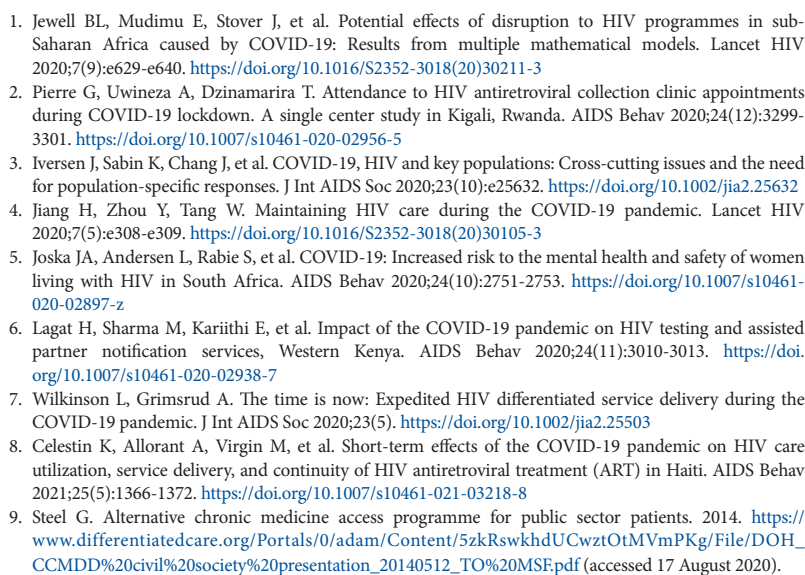
Saharan Africa caused by COVID-19: Results from multiple mathematical models. Lancet HIV 2020;7(9):e629-e640. https://doi.org/10.1016//2352-3018(20)30211-3

2. Pierre G, Uwineza A, Dzinamarira T. Attendance to HIV antiretroviral collection clinic appointments during COVID-19 lockdown. A single center study in Kigali, Rwanda. AIDS Behav 2020;24(12):32993301. https://doi.org/10.1007/s10461-020-02956-5

3. Iversen J, Sabin K, Chang J, et al. COVID-19, HIV and key populations: Cross-cutting issues and the need for population-specific responses. J Int AIDS Soc 2020;23(10):e25632. https://doi.org/10.1002/jia2.25632 4. Jiang H, Zhou Y, Tang W. Maintaining HIV care during the COVID-19 pandemic. Lancet HIV 2020;7(5):e308-e309. https://doi.org/10.1016/S2352-3018(20)30105-3

5. Joska JA, Andersen L, Rabie S, et al. COVID-19: Increased risk to the mental health and safety of women living with HIV in South Africa. AIDS Behav 2020;24(10):2751-2753. https://doi.org/10.1007/s10461020-02897-z

6. Lagat H, Sharma M, Kariithi E, et al. Impact of the COVID-19 pandemic on HIV testing and assisted partner notification services, Western Kenya. AIDS Behav 2020;24(11):3010-3013. https://do org/10.1007/s10461-020-02938-7

7. Wilkinson L, Grimsrud A. The time is now: Expedited HIV differentiated service delivery during the COVID-19 pandemic. J Int AIDS Soc 2020;23(5). https://doi.org/10.1002/jia2.25503

8. Celestin K, Allorant A, Virgin M, et al. Short-term effects of the COVID-19 pandemic on HIV care utilization, service delivery, and continuity of HIV antiretroviral treatment (ART) in Haiti. AIDS Behav utilization, service delivery, and continuity of HIV antiretroviral
2021;25(5):1366-1372. https://doi.org/10.1007/s10461-021-03218-8

9. Steel G. Alternative chronic medicine access programme for public sector patients. 2014. httpes/

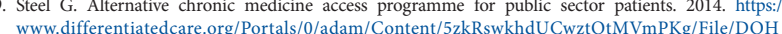
CCMDD\%20civil\%20society\%20presentation_20140512_TO\%20MSFpdf (accessed 17 August 2020).

S Afr Med J 2021;111(8):698. https://doi.org/10.7196/SAMJ.2021.v111i8.15864 